\title{
Plant Diversity of Gadchiroli district of Maharashtra, India: A brief survey
}

\author{
Suresh Jagtap* and Sourav Mukherjee \\ Bharati Vidyapeeth University, Interactive Research School for Health Affairs, Herbal Biotechnology Department, Katraj, Pune-Satara Road, Pune, \\ Pin-411 043, Maharashtra, India \\ * Corresponding author. Email: jagtapsd@gmail.com
}

\begin{abstract}
A qualitative survey was carried out during 2005- 2010 of all indigenous and naturalized plants of Gadchiroli district, Maharashtra, India. There are 237 species belonging to 184 genera and 73 families which are listed in this paper. Out of 73 families listed, 63 belong to dicotyledonae and 10 belong to monocotyledonae. Dominant families were Fabaceae (21 genera), Acanthaceae (12 genera) followed by Asteracece ( 9 genera). The nomenclature system followed in this paper is up to date. Three plants (1.63\%) listed in this paper come under Vulnerable (VU) and two (1.09\%) comes under Lower Risk (LR) categories. Eleven genera (5.98\%) are reported to be endemic to Maharashtra state of India. Only one plant species (Agave americana var. americana) was not native of the area and is cultivated. Voucher specimens of the representative plants are submitted at Medicinal Plants Conservation Center (MPCC), Pune, Maharashtra, and the voucher number is provided along with names of plants.
\end{abstract}

\section{INTRODUCTION}

In recent years, comprehensive floristic accounts have been published for various states, districts and regions of India (Kumar et al. 2007). However, for Gadchiroli district, no taxonomical study of the available plants has been carried so far, although few ethnobotanical studies are previously reported.

Major portion of the study area is covered with forestand the chief vegetation type is dry-deciduous (Tewari 1994; Tiwari and Padhye 1993) where teak and bamboo were found to be dominant elements. Many of the plants listed in this paper are utilized for medicinal purposes by the local tribal people, for example tree like Butea monosperma, Lannea coramandelica, Holarrhena pubescens, Pterocarpus marsupium, Soymida febrifuga, shrubs like Ceriscoides turgida, Gardenia resinifera, and herbs like Andrographis paniculata, Cullen corylifolia, Curculigo orchioides and Orthosiphon rubicundus. Summarily, the study aims to provide a list of plants occurring in Gadchiroli district of Maharashtra state of India.

\section{MATERIALS AND METHODS}

Present work is the outcome of 11 several seasonal field tours conducted during 2005- 2010. In every tour, attempts were made to collect speciments from all the areas of the district. Plants specimens were collected, dried, pressed and were identified with the help of published local flora, books (Cooke 1903; 1908; Sharma et al. 1996; Singh et al. 2001; Singh and Karthikeyan 2001) (Nagpur, Chandrapur) and by consulting the BSI western circle herbarium specimens. Plant specimens are deposited in the herbarium of Medicinal Plant Conservation Center, Pune (MPCC), Maharashtra under the first author's name.

Gadchiroli District, is situated in the South-eastern corner of Maharashtra, and is bounded by Chandrapur district in the west, Gondia District in the north, Chhatisgarh state in the east, and Andhra Pradesh state in the south and southwest (Figure 1). Gadchiroli district lies between $19^{\circ} 31^{\prime} \& 19^{\circ} 45^{\prime} \mathrm{N}$ latitude and $80^{\circ} 15^{\prime} \& 80^{\circ} 45^{\prime} \mathrm{E}$ longitude (Tiwari 1994). Total land cover of the study area is $14412.0 \mathrm{sq}$. km. Out of which, nearly $78.4 \%$ (i.e. 11694.0 sq. km. ) is covered by reserve or protected forest. Dhanora, Etapalli, Aheri and Sironcha talukas in the eastern part of the district are covered by forest. Remaining $1.55 \%$ land of the total area is wasteland. The area receives an annual rainfall of nearly $1510 \mathrm{~mm}$ (http://nagpur.nic.in/
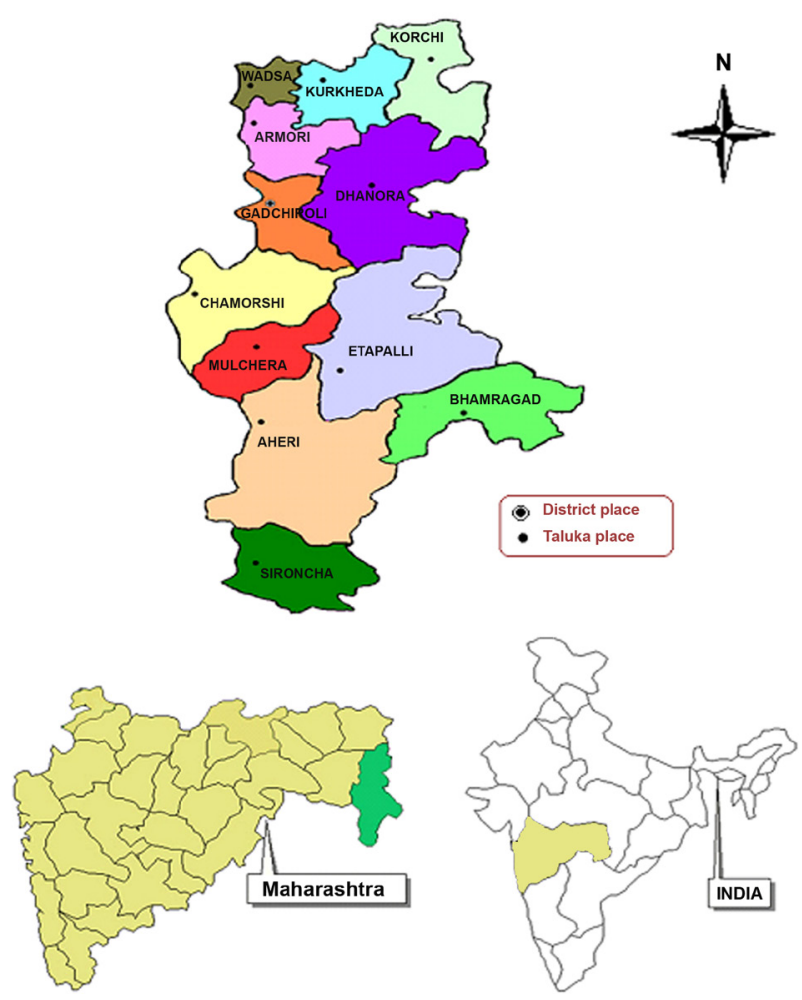

FIGURE 1. Map showing study area at Gadchiroli district of Maharashtra, India. 
divisionalcommissioner/Dist_info/GAD.html). The main river basin of the district is the Godavari, which flows west to east and forms the southern boundary of the district. Gadchiroli district has prominent hills like Bhamragad, Tipagad, Palasgad and Surjagad area (Tiwari 1994) and is inhabited by tribes such as the Halbi, Gond, Pawra, Bhil (Tiwari and Padhye 1993).

Main text of the research paper is table number 1 which gives floristic components of Gadchiroli. The botanical names of plants are given under family names (arranged alphabetically) followed by distribution, local names and the voucher specimen number under which the specimens are deposited at the MPCC.

\section{RESULTS AND DISCUSSION}

\section{Species diversity}

During the study, as many as 237 species of 184 genera belonging to 73 families (Table 1) were recorded. Out of these families, 10 belong to monocotyledonae (13.7 $\%$ ) and 63 are from dicotyledonous plant families (86.3 $\%$ dicotyledones). The families with highest number of species were Fabaceae (21 genera; $11.41 \%$ ), Acanthaceae (12 genera; $6.52 \%$ ), Asteracece (9 genera; $4.89 \%$ ), Euphorbiaceae and Rubiaceae (8 genera; $4.35 \%$ ) followed by Convolvulaceae, Cucurbitaceae, Lamiaceae ( 6 genera each; $3.26 \%$ ), together represented $30.43 \%$ of all identified genera. Amongst monocotyledonous plants, Liliaceae represented maximum number of genera $(4 ; 2.17 \%)$ followed by Araceae, Poaceae and Zingiberaceae (3 each; $1.63 \%)$. Thirty two $(32 ; 43.8 \%)$ families were represented by single genus and single species in the study area. In our sample most of the species (99.5\%) were native to the region and only one plant was cultivated (Agave americana var. americana). Tree species of the study area plays a vital role for day to day life of the tribal communities. Teak, bamboo being predominant components of the forest and are regularly used by the tribes for making home. Many plants listed here are used by local tribes for different purposes including medicinal aspect (Tewari 1994; Tiwari and Padhye 1993). Distribution of these listed plants were documented as abundant, common, cultivated, occasional based on the number of times they were encountered during exploration.

\section{Status Determination of taxa}

To assign status of distribution pattern of a taxa, the flora of Maharashtra by singh et. al (2001) and Singh and Karthikeyan (2001) were referred. Eleven plants (5.98\%) which are endemic to Maharashtra state of India are reported from the study area, viz., Alysicarpus belgaumensis, Amorphophallus commutatus, Arisaema murrayi, Barleria gibsoni, Blumea eriantha, Boswellia serrata, Canscora decurrens, Crotolaria filipes var. trichophor, Curcuma pseudomontana, Gantelbua urens, Haplanthodes verticillatus. Three taxa (1.63\%) reported form the study area fall under vulnerable category (VU) like Alysicarpus belgummensis, Arisaema murrayi, Barleria gibsonii, and two (1.09\%) fall under lower risk (LR) category like Amorphophallus commutatus and Haplanthodes verticillatus (Singh et al. 2001; Singh and Karthikeyan 2001).

Thus this paper gives superficial idea about floristic composition of Gadchiroli district.

TABLE 1. List of plants their families, distribution, local name and herbarium number.

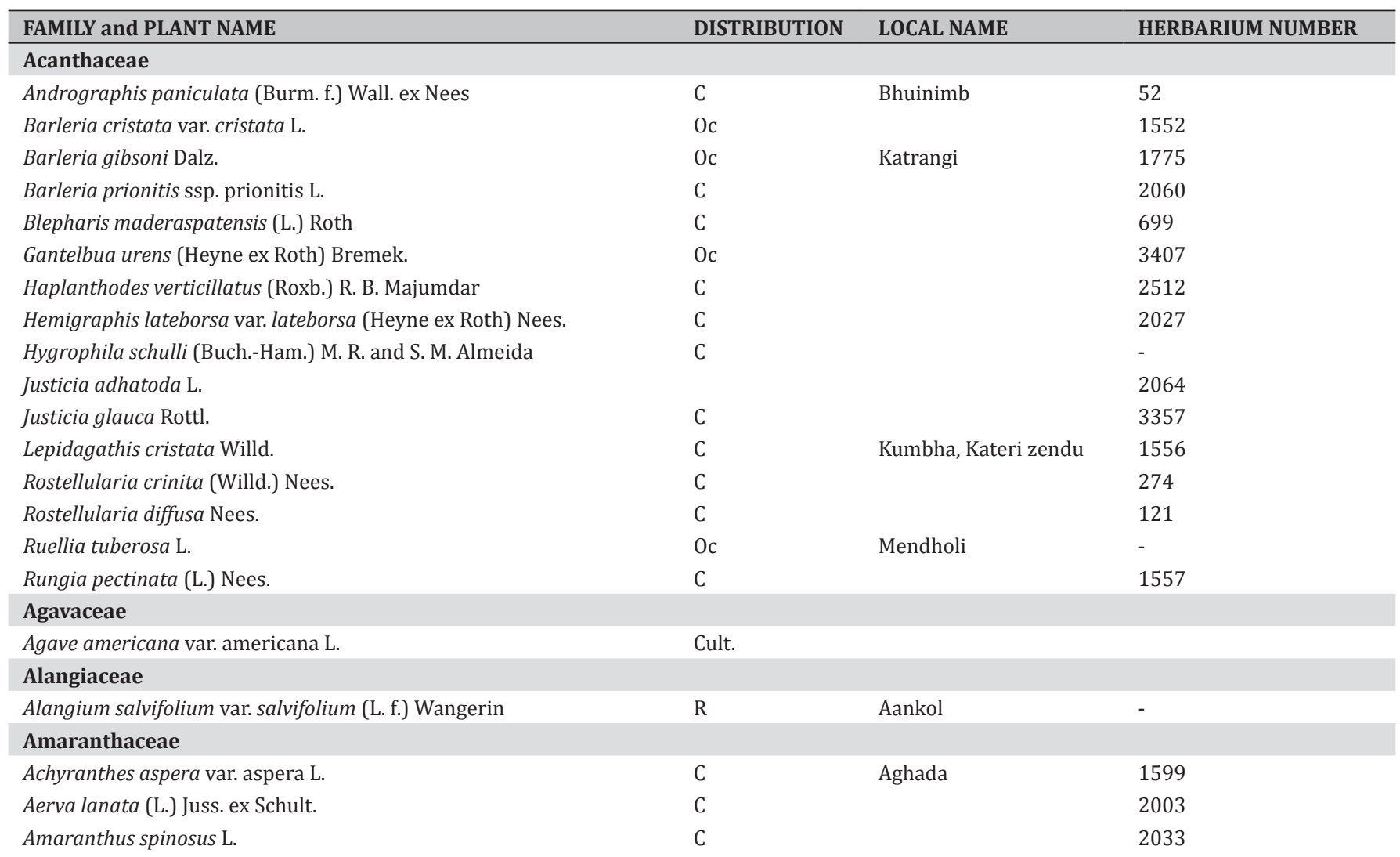


TABle 1. Continued.

\begin{tabular}{|c|c|c|c|}
\hline FAMILY and PLANT NAME & DISTRIBUTION & LOCAL NAME & HERBARIUM NUMBER \\
\hline \multicolumn{4}{|l|}{ Anacardiaceae } \\
\hline Buchanania cochinchinensis (Lour.) Almeida & $\mathrm{C}$ & Char & 2023 \\
\hline Lannea coromandelica (Houtt.) Merr. & $\mathrm{C}$ & Mowai & - \\
\hline Mangifera indica $\mathrm{L}$. & $\mathrm{C}$ & Amba & - \\
\hline Semecarpus anacardium var. anacardium L. f. & $\mathrm{R}$ & Biba & 863 \\
\hline \multicolumn{4}{|l|}{ Apocynaceae } \\
\hline Wrightia tinctoria var. rothii (G. Don) Ngan & $\mathrm{C}$ & Kadu kuda & 152 \\
\hline \multicolumn{4}{|l|}{ Araceae } \\
\hline Amorphophallus commutatus Engl. & $\mathrm{C}$ & & - \\
\hline Arisaema murrayi Hook. & 0 & Var & - \\
\hline Colocasia esculanta Schott & Oc & Kochai & 1891 \\
\hline \multicolumn{4}{|l|}{ Arecaceae } \\
\hline \multicolumn{4}{|l|}{ Asclepiadaceae } \\
\hline Calotropis gigantea (L.) Ait. & Oc & Rui & 2022 \\
\hline Calotropis procera (Ait.) R. Br. & Oc & Rui & - \\
\hline Cryptolepis buchanani Roem. and Schult. & $\mathrm{C}$ & & 261 \\
\hline Pergularia daemia (Forssk.) Choiv. & Oc & & 784 \\
\hline \multicolumn{4}{|l|}{ Asteraceae } \\
\hline Ageratum conyzoides L. & $\mathrm{C}$ & & 1513 \\
\hline Bidens biternata (Lour.) Merr. and Sherff. & $\mathrm{C}$ & & 187 \\
\hline Blumea eriantha DC. & $\mathrm{C}$ & Tambati & 1183 \\
\hline Eclipta prostata (L.) L. & Oc & & 2714 \\
\hline Elephantopus scaber L. & $\mathrm{C}$ & Jata shankar & 77 \\
\hline Emilia sonchifolia (L.) DC. & $\mathrm{C}$ & & 1567 \\
\hline Lagascea mollis Cav. & $\mathrm{C}$ & & 2741 \\
\hline Sphaeranthus indicus L. & $\mathrm{Ab}$ & Godhurli & - \\
\hline \multicolumn{4}{|l|}{ Balanitaceae } \\
\hline Balanites aegyptiaca (L.) Del. & Oc & & - \\
\hline \multicolumn{4}{|l|}{ Balsaminaceae } \\
\hline Impatiens balsamina var. balsamina L. & $\mathrm{R}$ & & 2057 \\
\hline \multicolumn{4}{|l|}{ Bignonaceae } \\
\hline Stereospermum chelenoides (L. f.) DC. & $\mathrm{R}$ & Parad & 90 \\
\hline Stereospermum colais (Buch.-Ham. ex Dillw.) Mabberley. & Oc & Surya & 2081 \\
\hline Dolichandrone falcata (Wall. ex. DC.) Seem. & $\mathrm{C}$ & Medshingi & - \\
\hline \multicolumn{4}{|l|}{ Bombacaceae } \\
\hline Bombax ceiba L. & Oc & Semal & - \\
\hline \multicolumn{4}{|l|}{ Brassicaceae } \\
\hline Brassica nigra (L.) Koch & Oc & & 1323 \\
\hline \multicolumn{4}{|l|}{ Burseraceae } \\
\hline Boswellia serrata Roxb. ex Colebr. & Oc & Salai & - \\
\hline \multicolumn{4}{|l|}{ Caesalpinaceae } \\
\hline Bauhinia purpurea L. & $\mathrm{C}$ & & 707 \\
\hline Bauhinia racemosa Lam. & $\mathrm{C}$ & Chehra & 903 \\
\hline Bauhinia tomentosa $\mathrm{L}$. & Oc & & 2622 \\
\hline Cassia fistula L. & Oc & Bahawa & - \\
\hline Cassia pumila Lam. & $\mathrm{C}$ & - & 1572 \\
\hline Cassia tora $\mathrm{L}$. & $\mathrm{C}$ & Tarota & 1107 \\
\hline Tamarindus indica L. & $\mathrm{C}$ & Chinch & 2012 \\
\hline \multicolumn{4}{|l|}{ Campanulaceae } \\
\hline Wahlenbergia erecta (Roth ex R and S.) Moeliona and Tuyn & Oc & & - \\
\hline \multicolumn{4}{|l|}{ Capparidaceae } \\
\hline Capparis zeylanica L. & Oc & Warakali & 777 \\
\hline \multicolumn{4}{|l|}{ Celastraceae } \\
\hline
\end{tabular}


Table 1. Continued.

\begin{tabular}{|c|c|c|c|}
\hline FAMILY and PLANT NAME & DISTRIBUTION & LOCAL NAME & HERBARIUM NUMBER \\
\hline \multicolumn{4}{|l|}{ Cocholospermaceae } \\
\hline Cochlospermum religiosum (L.) Alst. & $\mathrm{C}$ & Ghongal & - \\
\hline \multicolumn{4}{|l|}{ Combrataceae } \\
\hline Anogeissus latifolia (Roxb. ex DC.) Wall. ex Guill. and Perr. & $\mathrm{C}$ & Dhawda & 2211 \\
\hline Calycopteris floribunda (Roxb.) Poir. & $\mathrm{Ab}$ & Jilbuli & 367 \\
\hline Combretum albidum G. Don & Fr & piwalwel & 106 \\
\hline Terminalia bellirica (Gaerten.) Roxb. & Oc & & - \\
\hline Terminalia chebula Retz. & Oc & ajan & 158 \\
\hline Terminalia elliptica Willd. & $\mathrm{C}$ & Ain & 731 \\
\hline \multicolumn{4}{|l|}{ Commelinaceae } \\
\hline Cyanotis cristata Don. & $\mathrm{C}$ & & 2600 \\
\hline Murdannia nudiflora Brenan & $\mathrm{C}$ & & 1591 \\
\hline Argyreia sericea Dalz. and Gibs. & Oc & & 1077 \\
\hline Cuscuta chinensis var. chinensis Lam. & Oc & & - \\
\hline Cuscuta reflexa Roxb. & $\mathrm{R}$ & & - \\
\hline Evolvulus alsinoides (L.) L. & $\mathrm{C}$ & Vishnu kranta & 2717 \\
\hline Ipomoea cairica (L.) Sweet & $\mathrm{C}$ & Ganbhowara & 3121 \\
\hline Ipomoea pes-tigrides L. & Oc & Bilukhoj & 122 \\
\hline Merremia gangetica (L.) Cufod. & $\mathrm{C}$ & & 2059 \\
\hline \multicolumn{4}{|l|}{ Cucurbitaceae } \\
\hline Coccinia grandis (L.) Voigt & $\mathrm{R}$ & Tondalvel, Rantondli & - \\
\hline Luffa acutangula (L.) Roxb. & Oc & Randodka & - \\
\hline Momordica dioica Roxb. ex Willd. & $\mathrm{C}$ & Katul & 735 \\
\hline Mukia maderaspatana (L.) Roem. & Oc & & 3154 \\
\hline Trichosanthes anguina L. & $\mathrm{C}$ & Indravan & - \\
\hline Trichosanthes cucumerina L. & $\mathrm{C}$ & Indravan & - \\
\hline \multicolumn{4}{|l|}{ Cyperaceae } \\
\hline Kyllinga brevifolia Rottb. & $\mathrm{C}$ & & - \\
\hline Kyllinga melanosperma Nees. & $\mathrm{C}$ & & \\
\hline Kyllinga nemoralis Nees. & & & 3191 \\
\hline Kyllinga tenuifolia Steud. & $\mathrm{C}$ & & 1148 \\
\hline \multicolumn{4}{|l|}{ Dioscoriaceae } \\
\hline Dioscorea belophylla Haines & Oc & Kadukanda & - \\
\hline Dioscorea bulbifera L. & $\mathrm{C}$ & Mataru vel & 906 \\
\hline \multicolumn{4}{|l|}{ Ebenaceae } \\
\hline Diospyros melanoxylon Roxb. & $\mathrm{C}$ & Tendu & 3217 \\
\hline Diospyros montana Roxb. & Oc & & 2336 \\
\hline \multicolumn{4}{|l|}{ Eriocaulaceae } \\
\hline Eriocaulon cinereum R.Br. & $\mathrm{C}$ & & 2580 \\
\hline Eriocaulon elenorae Fyson & $\mathrm{C}$ & & - \\
\hline \multicolumn{4}{|l|}{ Euphorbiaceae } \\
\hline Acalypha ciliata Forssk. & Oc & & 1125 \\
\hline Antidesma acidum Retz. & $\mathrm{R}$ & & 860 \\
\hline Bridelia hamiltoniana Wall. ex Muell-Arg. & $\mathrm{C}$ & Kudurshi & - \\
\hline Bridelia retusa (L.) Spr. & Oc & Kasai & 464 \\
\hline Cleistanthus collinus (Roxb.) Benth.ex Hook. f. & $\mathrm{Ab}$ & Garari & - \\
\hline Emblica officinalis Gaertn. & Oc & Aawarun / Amla & 1533 \\
\hline Euphorbia geniculata Orteg. & $\mathrm{C}$ & & 2504 \\
\hline Euphorbia hirta L. & $\mathrm{C}$ & Kachara & 1507 \\
\hline Euphorbia rosea Retz. & $\mathrm{C}$ & & 2915 \\
\hline Mallotus philippinesis (Lam.) Muell.-Arg. & $\mathrm{R}$ & Shendri & 927 \\
\hline Phyllanthus urinaria L. & $\mathrm{C}$ & Bhui awli & 702 \\
\hline Phyllanthus virgatus Forst. $\mathrm{f}$. & Oc & Bhuiawali & 871 \\
\hline \multicolumn{4}{|l|}{ Fabaceae } \\
\hline
\end{tabular}


TABle 1. Continued.

\begin{tabular}{|c|c|c|c|}
\hline FAMILY and PLANT NAME & DISTRIBUTION & LOCAL NAME & HERBARIUM NUMBER \\
\hline Alysicarpus hamosus Edgew. & $\mathrm{C}$ & & 24 \\
\hline Alysicarpus tetragonolobus Edgew. & $\mathrm{C}$ & & 119 \\
\hline Butea monosperma var. monosperma Roxb. ex Willd. & $\mathrm{C}$ & Palas & 2904 \\
\hline Butea superba Roxb. ex Willd. & Oc & Palaswel & - \\
\hline Cajanus platycarpus (Benth.) Vander der Maesen & $\mathrm{C}$ & Supulsheng & 3403 \\
\hline Cajanus scarabaeoides (L.) du-Petit-Thours & $\mathrm{C}$ & & 67 \\
\hline Crotalaria filipes var. trichophora (Bth. ex Baker) T. Cooke & Oc & & 2631 \\
\hline Crotalaria hebecarpa (DC.) Rudd & $\mathrm{C}$ & & 3156 \\
\hline Crotalaria hirsuta Willd. & Oc & Tichakali & 40 \\
\hline Cullen corylifolia (L.) Medik. & $\mathrm{R}$ & & 946 \\
\hline Dalbergia lanceolaria ssp. lanceolaria L. f. & $\mathrm{C}$ & Shevdor & - \\
\hline Desmodium gangeticum (L.) DC. & $\mathrm{C}$ & & 87 \\
\hline Desmodium triflorum (L.) DC. & $\mathrm{C}$ & Chiputi & 1570 \\
\hline Desmodium velutinum (Willd.) DC. & $\mathrm{C}$ & Chipadi & 145 \\
\hline Indigofera trita var. trita $\mathrm{L}$. & $\mathrm{C}$ & Ranmethi & 3423 \\
\hline Macrotyloma uniflorum (Lam.) Verdc. & Oc & & 709 \\
\hline Pongamia pinnata (L.) Pierre & Oc & Karanj & - \\
\hline Pterocarpus marsupium var. marsupium Roxb. & $\mathrm{Ab}$ & Biwala & 755 \\
\hline Teramnus labialis (L. f.) Spreng. & $\mathrm{C}$ & Ranudid, Tipani & 2629 \\
\hline Teramnus mollis Bth. & Oc & & 3166 \\
\hline Vigna aconitifolia (Jacq.) Marechal & $\mathrm{C}$ & & 89 \\
\hline Vigna dalzelliana (0. Ketz.) Verdc & $\mathrm{C}$ & Rangavar & 1558 \\
\hline Zornia gibbosa Span. & $\mathrm{Ab}$ & & 1571 \\
\hline \multicolumn{4}{|l|}{ Flacourtiaceae } \\
\hline Caeseria graveolens Dalz. & $\mathrm{R}$ & Kukuddawana & - \\
\hline Caeseria tomentosa Roxb. & $\mathrm{R}$ & & - \\
\hline Flacourtia indica (Burm. f.) Merr. & $\mathrm{C}$ & Kakai & - \\
\hline \multicolumn{4}{|l|}{ Gentianaceae } \\
\hline Canscora decurrens Dalz. & Oc & & 942 \\
\hline Canscora decussata (Roxb.) A.schult and J.H.schult & Oc & & 1555 \\
\hline Exacum pumilum Griseb. & $\mathrm{C}$ & & 3363 \\
\hline \multicolumn{4}{|l|}{ Hypoxidaceae } \\
\hline Curculigo orchioides Geartn. & Oc & Dukkarkand, kali Musli & 1142 \\
\hline \multicolumn{4}{|l|}{ Lamiaceae } \\
\hline Hyptis suaveolens (L.) Poit. & Oc & & 2202 \\
\hline Lavendula bipinnata var. rothina 0 . Ketz. & $\mathrm{C}$ & & 940 \\
\hline Leucas aspera (Willd.) Link. & $\mathrm{C}$ & & 2062 \\
\hline Leucas stelligera Wall. & $\mathrm{C}$ & & 1935 \\
\hline Leucas stricta Bth. & $\mathrm{C}$ & & 195 \\
\hline Ocimum americanum $\mathrm{L}$. & $\mathrm{C}$ & & 2005 \\
\hline Ocimum basilicum var. thyrsiflora Bth. & $\mathrm{C}$ & & 1114 \\
\hline Ocimum tenuiflorum $\mathrm{L}$. & Oc & & 3431 \\
\hline Orthosiphon rubicundus (D. Don) Benth. & $\mathrm{C}$ & & 867 \\
\hline Tectona grandis L. f. & $\mathrm{C}$ & sag & - \\
\hline \multicolumn{4}{|l|}{ Lecythidaceae } \\
\hline Careya arborea Roxb. & Oc & Kumbha & - \\
\hline \multicolumn{4}{|l|}{ Leeaceae } \\
\hline Leea indica (Burm. f.) Merr. & Oc & & 3161 \\
\hline \multicolumn{4}{|l|}{ Liliaceae } \\
\hline Asparagus racemosus var. racemosus Willd. & A & Marbat & 1558 \\
\hline Chlorophytum laxum R. Br. & $\mathrm{C}$ & Musali & 667 \\
\hline Chlorophytum tuberosum Baker & $\mathrm{C}$ & Safed musali & 668 \\
\hline Gloriosa superba L. & Oc & Karkari & 1523 \\
\hline Scilla hyacinthina Mc Bride & $\mathrm{C}$ & & 2006 \\
\hline \multicolumn{4}{|l|}{ Loganiaceae } \\
\hline Mitreola petiolata (J.F.Gmel.)Torr.and A.Grey & 0 & & 61 \\
\hline \multicolumn{4}{|l|}{ Loranthaceae } \\
\hline Dendrophthoe falcata var. falcata (L. f.) Etting & Oc & & - \\
\hline
\end{tabular}


TABle 1. Continued.

\begin{tabular}{|c|c|c|c|}
\hline FAMILY and PLANT NAME & DISTRIBUTION & LOCAL NAME & HERBARIUM NUMBER \\
\hline \multicolumn{4}{|l|}{ Lythraceae } \\
\hline Ammannia auriculata Willd. & Oc & & 133 \\
\hline Lagerstromea parviflora Roxb. & Oc & Sehna & 265 \\
\hline Woodfordia fruticosa (L.) Kurz & $\mathrm{C}$ & Jilbuli & 54 \\
\hline \multicolumn{4}{|l|}{ Malvaceae } \\
\hline Sida acuta Burm. f. & $\mathrm{C}$ & & 118 \\
\hline Sida rhombifolia L. & $\mathrm{C}$ & & 2408 \\
\hline Urena lobata L. & $\mathrm{C}$ & & 1771 \\
\hline \multicolumn{4}{|l|}{ Martyniaceae } \\
\hline Martynia annua L. & Oc & Vagh nakhi & 2938 \\
\hline \multicolumn{4}{|l|}{ Melastomataceae } \\
\hline Osbeckia muralis Naud. & Oc & & - \\
\hline Azadirachta indica A. Juss. & $\mathrm{R}$ & & - \\
\hline Chlroxylon swietenia DC. & $\mathrm{R}$ & Mhasawad & - \\
\hline Soymida febrifuga (Roxb.) A. Juss. & $\mathrm{C}$ & Rohan & 2618 \\
\hline \multicolumn{4}{|l|}{ Menispermaceae } \\
\hline Cocculus hirsutus (L.) Theob. & Oc & vasanvel & 105 \\
\hline \multicolumn{4}{|l|}{ Mimosaceae } \\
\hline Acacia catechu (L. f.) Willd. & Oc & Khair & 1115 \\
\hline Acacia leucophloea (Roxb.) Willd. & $\mathrm{C}$ & Hivar & 2245 \\
\hline Acacia torta (Roxb.) Craib & $\mathrm{C}$ & Chilati & - \\
\hline Albizia lebbeck var. lebbeck (L.) Benth. & Oc & & - \\
\hline Albizia odoratissima var. subhedarii (L.) Benth. & $\mathrm{C}$ & Chichva & 772 \\
\hline Albizia procera (Roxb.) Benth. & Oc & & - \\
\hline Xylia xylocarpa(Roxb.) Taub. & $\mathrm{R}$ & Surya & \\
\hline \multicolumn{4}{|l|}{ Moraceae } \\
\hline Ficus benghalensis L. & $\mathrm{R}$ & & - \\
\hline Ficus racemosa $\mathrm{L}$. & $\mathrm{R}$ & & - \\
\hline Ficus religiosa L. & Oc & Pimpal & - \\
\hline \multicolumn{4}{|l|}{ Nyctaginaceae } \\
\hline Boerhavia repens var. diffusa L. & $\mathrm{C}$ & & 2509 \\
\hline \multicolumn{4}{|l|}{ Olacaceae } \\
\hline Olax psittacorum (Willd.) Vahl. & Oc & Haradphari & 656 \\
\hline \multicolumn{4}{|l|}{ Oleaceae } \\
\hline Schrebera swietenioides Roxb. & Oc & Mokha & - \\
\hline \multicolumn{4}{|l|}{ Onagraceae } \\
\hline Ludwigia octovalis var. sessiliflora (Micheli.) Raven & Oc & & 2705 \\
\hline \multicolumn{4}{|l|}{ Opiliaceae } \\
\hline Cansjera rheedii Gmel. & Oc & Haradfari & - \\
\hline \multicolumn{4}{|l|}{ Orchidaceae } \\
\hline Rhynchostylis retusa $\mathrm{Bl}$. & Oc & Kanda & - \\
\hline Vanda tessellata (Roxb.) Hook. & Oc & Banda & 587 \\
\hline Vanda testacea Reich. f. & Oc & & 383 \\
\hline \multicolumn{4}{|l|}{ Oxalidaceae } \\
\hline Biophytum candolleanum Wight & $\mathrm{C}$ & Lajalu & 75 \\
\hline Biophytum reinwardtii (Zucc.) Klotz. & $\mathrm{C}$ & Lajalu & 2431 \\
\hline \multicolumn{4}{|l|}{ Pedaliaceae } \\
\hline Sesamum orientale L. & $\mathrm{C}$ & Til & 3411 \\
\hline \multicolumn{4}{|l|}{ Periplocaceae } \\
\hline Hemidesmus indicus (L.) Schult. & $\mathrm{C}$ & & 494 \\
\hline Poaceae & & & \\
\hline Cymbopogon martinii Wats. & $\mathrm{C}$ & & 2647 \\
\hline Cynadon dactylon Pers. & $\mathrm{C}$ & & - \\
\hline
\end{tabular}


TABle 1. Continued.

\begin{tabular}{|c|c|c|c|}
\hline FAMILY and PLANT NAME & DISTRIBUTION & LOCAL NAME & HERBARIUM NUMBER \\
\hline \multicolumn{4}{|l|}{ Rhamnaceae } \\
\hline Ventilago denticulata Willd. & Oc & Popati & 101 \\
\hline Ziziphus caracutta Roxb. & Oc & Guti & 856 \\
\hline Ziziphus mauritiana Lam. & $\mathrm{C}$ & Bor & 904 \\
\hline Ziziphus oenoplia (L.) Mill. & Oc & - & 63 \\
\hline \multicolumn{4}{|l|}{ Rubiaceae } \\
\hline Catunaregam spinosa (Thunb.) Tirvengadum & Oc & Ghera & - \\
\hline Ceriscoides turgida (Roxb.) Tirveng. & $\mathrm{R}$ & Phedra pandra & - \\
\hline Gardenia latifolia Soland. & Oc & Kudmodi & - \\
\hline Gardenia resinifera Roth. & $\mathrm{C}$ & Mali, Jondhurli & 2206 \\
\hline Haldina cordifolia (Roxb.) Rasad. & Oc & & 2510 \\
\hline Ixora pavetta Andr. & $\mathrm{R}$ & Lokhandi & 2621 \\
\hline Mitragyna parvifolia (Roxb.) Korth. & $\mathrm{C}$ & Kalam & 957 \\
\hline Spermacoce articularis L. f. & Oc & & 3105 \\
\hline Tamilnadia uliginosa (Retz.) Tirveng. and Sastre. & $\mathrm{R}$ & Kala phedra & - \\
\hline \multicolumn{4}{|l|}{ Rutaceae } \\
\hline Aegle marmalos (L.) Corr. & $\mathrm{R}$ & & - \\
\hline \multicolumn{4}{|l|}{ Sapindaceae } \\
\hline Schleichera oleosa (Lour.) Oken & Oc & Kojab & 386 \\
\hline \multicolumn{4}{|l|}{ Sapotaceae } \\
\hline Madhuca longifolia var. longifolia (Koen.) Mac Bride & $\mathrm{Ab}$ & Mahuwa / Mowa & - \\
\hline \multicolumn{4}{|l|}{ Scrophulariaceae } \\
\hline Bacopa monnieri (L.) Penn. & $\mathrm{R}$ & & 959 \\
\hline Buchnera hispida Buch.-Ham. ex D. Don & Oc & & 2067 \\
\hline Lindernia ciliata (Colsm.) Penn. & $\mathrm{C}$ & & 68 \\
\hline Lindernia viscosa (Horhem.) Boldingh & $\mathrm{C}$ & & 1817 \\
\hline Sopubia delphinifolia var. delphinifolia (L.) G. Don & Oc & & 2579 \\
\hline Striga densiflora (Benth.) Benth. & Oc & Agaya & 2641 \\
\hline \multicolumn{4}{|l|}{ Smilacaceae } \\
\hline Smilax perfoliata Lour. & Oc & Sherdira & 2473 \\
\hline \multicolumn{4}{|l|}{ Solanaceae } \\
\hline Datura metel L. & Oc & & - \\
\hline Physalis minima L. & $\mathrm{C}$ & & 2507 \\
\hline Solanum virginianum L. & $\mathrm{C}$ & Bhuiringni & 2403 \\
\hline \multicolumn{4}{|l|}{ Sterculiaceae } \\
\hline Eriolaena quinquelocularis (Wight and Arn.) Wight & $\mathrm{C}$ & Bothi & 3188 \\
\hline Helicteres isora $\mathrm{L}$. & $\mathrm{C}$ & Atai & 60 \\
\hline Sterculia urens Roxb. & Oc & & - \\
\hline \multicolumn{4}{|l|}{ Tiliaceae } \\
\hline Corchorus olitorius L. & $\mathrm{C}$ & Chuch & 30 \\
\hline Corchorus tridens L. & $\mathrm{C}$ & & 3164 \\
\hline Grewia damine Gaertn. & $\mathrm{C}$ & & 864 \\
\hline Grewia tiliifolia Vahl. & Oc & Dhaman & 856 \\
\hline \multicolumn{4}{|l|}{ Verbenaceae } \\
\hline Clerodendrum serratum (L.) Moon. & $\mathrm{R}$ & Pimphar / Pinkphar & 936 \\
\hline Symphorema involucratum Roxb. & $\mathrm{R}$ & & - \\
\hline Vitex negundo L. & $\mathrm{C}$ & & 2624 \\
\hline \multicolumn{4}{|l|}{ Violaceae } \\
\hline Hybanthus enneaspermus (L.) F. v. Muell. & $\mathrm{C}$ & Lendoli & 3178 \\
\hline \multicolumn{4}{|l|}{ Vitaceae } \\
\hline Ampelocissus latifolia (Roxb.) Planch. & Oc & Dokel & 2010 \\
\hline Cissus repanda Vahl & $\mathrm{C}$ & Dokel vel & 706 \\
\hline \multicolumn{4}{|l|}{ Zinziberaceae } \\
\hline Costus speciosus Sm. & Oc & Harduli, Kevkand & - \\
\hline Curcuma pseudomontana Grahm. & $\mathrm{C}$ & Ranhalad & - \\
\hline Zingiber cernuum Dalz. & $\mathrm{R}$ & Ran ale & - \\
\hline
\end{tabular}


ACKNOWLEDGMENTS: The authors are grateful to Prof. P K Ranjekar, Director IRSHA, Bharati Vidyapeeth Deemed University, Pune for giving the opportunity to work.

\section{LITERATURE CITED}

Cooke, T. 1903. The Flora of The Presidency of Bombay volume 1. Taylor and Francis, London. $645 \mathrm{p}$.

Cooke, T. 1908. The Flora of The Presidency of Bombay volume 2. Taylor and Francis, London. 1083 p.

NOAA. 2012. Nagpur District Office. Version 2012.2. Electronic database accessible at http://nagpur.nic.in/divisionalcommissioner/Dist info/GAD.html. Captured on 22 January 2012.

Kumar, P., J.S. Jalal and G.S. Rawat. 2007. Orchidaceae, Chotanagpur, state of Jharkhand, India. Check List 3(4): 297-304.

Sharma, B.D., S. Karthikeyan and N.P. Singh. 1996. Flora of Maharashtra State (Flora of India Series 2): Monocotyledones. Calcutta: Botanical Survey of India. 789 p.

Singh, N.P. and S. Karthikeyan. 2001. Flora of Maharashtra State (Flora of India Series 2): Dicotyledones, volume 1. Calcutta: Botanical Survey of India. $882 \mathrm{p}$.
Singh, N.P., P. Lakshminarasimhan, S. Karthikeyan and P.V. Prasanna. 2001 Flora of Maharashtra State (Flora of India Series 2): Dicotyledones, volume 2. Calcutta: Botanical Survey of India. $1079 \mathrm{p}$.

Tiwari, VJ. 1994. Ethnobotanical survey of Halbi tribe of Chandrapur and Gadchiroli districts of Maharashtra State, India. Fitoterapia 66(4): 346-350.

Tiwari, V. J. and M. D. Padhye. 1993. Ethnobotanical study of Gond tribe of Chandrapur and Gadchiroli districts of Maharashtra state, India. Fitoterapia 64(1): 58-61.

RECEIVED: September 2011

ACCEPTED: November 2012

PUBLISHED ONLINE: February 2013

EDITORIAL RESPONSIBILITY: Angelo Gilberto Manzatto 